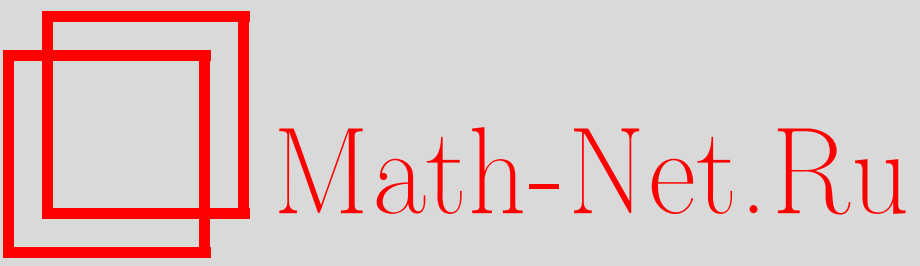

М. А. Ташкинов, Многоточечные моментные функции структурных свойств полидисперсных композитов, Вестн. Сам. гос. техн. ун-та. Сер. Физ.-мат. науки, 2011, выпуск 2(), 74-82

DOI: https://doi.org/10.14498/vsgtu942

Использование Общероссийского математического портала Math-Net.Ru подразумевает, что вы прочитали и согласны с пользовательским соглашением

http: //www. mathnet.ru/rus/agreement

Параметры загрузки:

IP : 54.205 .225 .156

26 апреля 2023 г., 02:42:30 
УДК 539.3

\section{МНОГОТОЧЕЧНЫЕ МОМЕНТНЫЕ ФУНКЦИИ СТРУКТУРНЫХ СВОЙСТВ ПОЛИДИСПЕРСНЫХ КОМПОЗИТОВ}

\section{М. А. Ташкинов}

Пермский государственный технический университет, 614990, Пермь, Комсомольский проспект, 29а.

E-mail: m.tashkinov@mail.ru

Приведена постановка стохастической краевой задачи теории упругости для двухфазных полидисперсных композитов. Описан метод её решения с использованием моментных бункиий структурных свойств высших порлдков. Представлен алгоритм построения моментных функций n-ного порядка для объёмных структур. Предложены аппроксимирующие зависимости для моментных функций. Приведены примеры вычисления моментных функиий высших порядков для полидисперсных структур.

Ключевые слова: композиты, моментные функиии, стохастическая краевая задача, трехмерные модели, случайная полидисперсная структура, аппроксимаиия.

Введение. Композиты широко применяются в инженерной практике благодаря уникальному сочетанию физико-механических свойств, возможности задания параметров материалов и предсказания его поведения. Разработка и внедрение таких материалов сопряжены с решением ряда задач, таких как выбор матрицы и наполнителя, учёт свойств каждого из компонентов для получения необходимых качеств материала, предсказание поведения материала под нагрузкой [1]. В механике композитов создано большое количество моделей. В отдельный класс можно выделить статистические модели, основанные на применении теории случайных функций. В большинстве их них для исследования структурных полей деформирования и расчета эффективных характеристик решается стохастическая краевая задача, уравнения и граничные условия которой содержат случайные величины. Наиболее широко используется дифференциальная постановка стохастической краевой задачи теории упругости [2], общая для большинства работ [3-6], когда коэффициенты дифференциального оператора являются случайными быстро осциллирующими кусочно-постоянными функциями координат (т. е. являются постоянными для каждого компонента и скачком меняют свои значения при переходе границы между компонентами) [7].

Один из способов решения стохастических краевых задач теории упругости связан с построением систем уравнений с использованием моментных функций структурных свойств, которые несут в себе информацию о геометрии структуры композитов. Получение таких моментных функций аналитическими и экспериментальными методами сопряжено с определенными трудностями. При аналитическом построении необходимо вводить гипотезы о характере взаимного расположения элементов структуры, нуждающиеся

Михаил Анатольевич Ташкинов, аспирант, каф. механики композиционных материалов и конструкций. 
в достаточном обосновании. Снижение точности оценок с увеличением порядка моментных функций обуславливает необходимость большего объёма исходной информации о структуре при построении моментных функций и многомерных законов распределения на основе экспериментальных данных. В результате оба метода оказываются недостаточно эффективными. В то же время информация, получаемая лишь моментными функциями низших порядков, недостаточна для описания структуры композитов при решении краевых задач в высших многоточечных приближениях. В связи с этим возникает необходимость в построении многоточечных моментных функций высших порядков, а также в получении аналитических выражений для них в явном виде.

В данной работе рассматриваются методы решения стохастической краевой задачи теории упругости в высших приближениях с использованием многоточечных моментных функций высших порядков, а также способы построения и аппроксимации моментных функций для синтезированных моделей матричных полидисперсных композитов со случайным образом распределенными объёмными включениями.

1. Стохастическая краевая задача теории упругости. Для математического описания композитов используется структурно-феноменологический подход, который состоит в том, что однородные физико-механические свойства компонентов структуры задаются с помощью общепринятых в механике феноменологических уравнений и критериев, а характеристики структурных полей деформирования и эффективные свойства композита вычисляются из решений краевых задач. Наибольшее распространение получил способ решения, в котором исходное стохастическое уравнение преобразуется в интегро-дифференциальное методом функций Грина с использованием тензора КельвинаСомильяны [2], а затем полученное уравнение относительно пульсаций перемещений решается методом последовательных приближений. При решении краевой задачи принимаются следующие гипотезы: физические и геометрические величины, описывающие свойства композита, считаются статистически однородными и эргодическими случайными полями; матрица и включения - однородные, упругие и изотропные; адгезия между компонентами по границам раздела предполагается идеальной; воздействие массовых сил на компоненты композитов не учитывается; геометрия и взаимное расположение элементов структуры предполагаются заданными и неизменяющимися в процессе деформирования, а сама среда обладает свойством макроскопической однородности.

С учётом принятых гипотез краевая задача теории упругости для композитов со случайной структурой записывается следующим образом:

$$
\begin{gathered}
\partial \sigma_{i j}(\vec{r}) / \partial x_{j}=0, \quad \varepsilon_{i j}(\vec{r})=0,5\left(U_{i, j}(\vec{r})+U_{j, i}(\vec{r})\right), \\
\sigma_{i j}(\vec{r})=C_{i j k l}(\vec{r}) \varepsilon_{k l}(\vec{r}), \\
\left.U_{i}(\vec{r})\right|_{\vec{r} \in \Gamma_{u}}=e_{i j} r_{j},
\end{gathered}
$$

где $e_{i j}$ - постоянный произвольно заданный симметричный тензор малых деформаций, $\vec{r}$ - радиус-вектор с компонентами $\left(x_{1}, x_{2}, x_{3}\right)$, граничные условия (2) заданы в перемещениях. 
Геометрия структуры двухфазного композита определяется с помощью индикаторной функции

$$
\lambda(\vec{r})= \begin{cases}1, & \vec{r} \in V_{I} \\ 0, & \vec{r} \in V_{M}\end{cases}
$$

где $V_{I}$ - объём, занимаемый включениями; $V_{M}$ - объём, занимаемый матрицей.

С помощью индикаторной функции поле структурных модулей упругости $C_{i j k l}(\vec{r})$ двухфазного композита записывается как

$$
C_{i j k l}(\vec{r})=\lambda(\vec{r}) C_{i j k l}^{I}+(1-\lambda(\vec{r})) C_{i j k l}^{M},
$$

где $C_{i j k l}^{I}$ и $C_{i j k l}^{M}$ - заданные модули упругости включений и матрицы соответственно. После операции осреднения получаем

$$
\left\langle C_{i j k l}(\vec{r})\right\rangle=p C_{i j k l}^{I}+(1-p) C_{i j k l}^{M},
$$

где $p=\langle\lambda(\vec{r})\rangle$ - объёмная доля включений. Предполагается, что индикаторная функция (3) обладает свойством эргодичности и статистической однородности, поэтому операцию математического осреднения можно проводить не по реализациям, а по объёму, т. е. для любых $\vec{r}_{\text {и }} \vec{r}_{1}$ имеем $\langle\lambda(\vec{r})\rangle=\left\langle\lambda\left(\vec{r}_{1}\right)\right\rangle$.

Введём величину пульсации индикаторной функции $\lambda^{\prime}(\vec{r})$ как $\lambda^{\prime}(\vec{r})=$ $=\lambda(\vec{r})-\langle\lambda(\vec{r})\rangle$ и запишем поля структурных модулей упругости и поля перемещений в виде суммы средней составляющей и пульсации:

$$
C_{i j k l}(\vec{r})=\left\langle C_{i j k l}(\vec{r})\right\rangle+C_{i j k l}^{\prime}(\vec{r}), \quad U_{m}(\vec{r})=\left\langle U_{m}(\vec{r})\right\rangle+U_{m}^{\prime}(\vec{r}) .
$$

При помощи преобразований, подробно описанных в [8], краевая задача сводится к интегро-дифференциальному уравнению, содержащему функцию Грина:

$$
\begin{array}{r}
\frac{\partial U_{i}^{\prime(\chi)}(\vec{r})}{\partial x_{j}}=\int_{V_{1}} \frac{\partial G_{i m}\left(\vec{r}, \vec{r}_{1}\right)}{\partial x_{j}} \frac{\partial}{\partial x_{1 n}}\left(\left(\left\langle C_{m n k l}\left(\vec{r}_{1}\right)\right\rangle+C_{m n k l}^{\prime}\left(\vec{r}_{1}\right)\right) e_{k l}+\right. \\
\left.+C_{m n k l}\left(\vec{r}_{1}\right) U_{k, l}^{\prime(\chi-1)}\left(\vec{r}_{1}\right)\right) d V_{1}
\end{array}
$$

где $G_{i m}\left(\vec{r}, \vec{r}_{1}\right)$ - функция Грина, $\chi$ - приближение, в котором решается задача. В случае первого приближения $\chi=1, U_{k, l}^{\prime(0)}\left(\vec{r}_{1}\right)=0$. Поля напряжений и деформаций определяются через закон Гука (1) и перемещения (4):

$$
\begin{gathered}
\varepsilon_{i j}^{\prime}(\vec{r})=0,5\left(U_{i, j}^{\prime}(\vec{r})+U_{j, i}^{\prime}(\vec{r})\right), \\
\sigma_{i j}^{\prime}(\vec{r})=\sigma_{i j}(\vec{r})-\left\langle\sigma_{i j}(\vec{r})\right\rangle=C_{i j m n}^{\prime}(\vec{r}) e_{m n}-\left\langle C_{i j m n}^{\prime}(\vec{r}) \varepsilon_{m n}^{\prime}(\vec{r})\right\rangle+C_{i j m n}(\vec{r}) \varepsilon_{m n}^{\prime}(\vec{r}) .
\end{gathered}
$$

Статистические характеристики полей напряжений и деформаций представляют собой моменты первого и второго порядка тензоров (5) и (6). Безусловные моменты $M_{i j \alpha \beta}^{(\sigma)}=\left\langle\sigma_{i j}^{\prime}(\vec{r}) \sigma_{\alpha \beta}^{\prime}(\vec{r})\right\rangle, M_{i j \alpha \beta}^{(\varepsilon)}=\left\langle\varepsilon_{i j}^{\prime}(\vec{r}) \varepsilon_{\alpha \beta}^{\prime}(\vec{r})\right\rangle$ характеризуют композит как макрооднородный материал, в то время как условные моменты являются характеристиками полей деформирования в компонентах - 
матрице $\left\langle\varepsilon_{i j}(\vec{r})\right\rangle_{M},\left\langle\sigma_{i j}(\vec{r})\right\rangle_{M}, T_{i j \alpha \beta}^{(\sigma)}=\left\langle\sigma_{i j}^{\prime}(\vec{r}) \sigma_{\alpha \beta}^{\prime}(\vec{r})\right\rangle_{M}, T_{i j \alpha \beta}^{(\varepsilon)}=\left\langle\varepsilon_{i j}^{\prime}(\vec{r}) \varepsilon_{\alpha \beta}^{\prime}(\vec{r})\right\rangle_{M}$ или включениях (записываются аналогично).

2. Моментные функции структурных свойств. Идея рассматриваемого метода заключается в том, что безусловные и условные моменты второго порядка (дисперсии) можно выразить через смешанные моменты типа $\left\langle\lambda^{\prime}\left(\vec{r}_{1}\right) \lambda^{\prime}\left(\vec{r}_{2}\right) \times\right.$ $\left.\times U_{m, n}^{\prime}\left(\vec{r}_{1}\right)\right\rangle,\left\langle\lambda^{\prime}\left(\vec{r}_{1}\right) \lambda^{\prime}\left(\vec{r}_{2}\right) U_{m, n}^{\prime}\left(\vec{r}_{1}\right) U_{\nu, \mu}^{\prime}\left(\vec{r}_{2}\right)\right\rangle$, которые, в свою очередь, содержат константы и моментные функции высших порядков индикаторной функции $\lambda(\vec{r})$ (см. [8]). При решении краевой задачи во втором приближении требуются моментные функции вплоть до пятого порядка.

Запишем выражение для моментной функции $n$-ного порядка:

$$
\begin{aligned}
& K\left(\vec{r}, \vec{r}_{1}, \ldots, \vec{r}_{n}\right)=\left\langle\lambda^{\prime}(\vec{r}) \lambda^{\prime}\left(\vec{r}_{1}\right) \cdots \lambda^{\prime}\left(\vec{r}_{n}\right)\right\rangle= \\
& =\left\langle(\lambda(\vec{r})-\langle\lambda(\vec{r})\rangle)\left(\lambda\left(\vec{r}_{1}\right)-\left\langle\lambda\left(\vec{r}_{1}\right)\right\rangle\right) \cdots\left(\lambda\left(\vec{r}_{n}\right)-\left\langle\lambda\left(\vec{r}_{n}\right)\right\rangle\right)\right\rangle= \\
& \quad=\left\langle(\lambda(\vec{r})-p)\left(\lambda\left(\vec{r}_{1}\right)-p\right) \cdots\left(\lambda\left(\vec{r}_{n}\right)-p\right)\right\rangle .
\end{aligned}
$$

Так как $\lambda(\vec{r})$ - статистически однородная и изотропная функция и $\left\langle\lambda\left(\vec{r}_{n}\right)\right\rangle=$ $=p=$ const, моментные функции зависят только от расстояний между рассматриваемыми точками $\left|\vec{r}_{m}-\vec{r}_{n}\right|$, т. е. искомую моментную функцию второго порядка (корреляционную функцию) можно представить в виде

$$
K_{\lambda}^{2}\left(\left|\vec{r}-\vec{r}_{1}\right|\right)=D_{\lambda}^{2} f_{\lambda}^{2}\left(\left|\vec{r}-\vec{r}_{1}\right|\right)
$$

где $f_{\lambda}^{2}\left(\left|\vec{r}-\vec{r}_{1}\right|\right)$ - нормированная корреляционная функция, $D_{\lambda}^{2}=\left\langle\left(\lambda^{\prime}(\vec{r})\right)^{2}\right\rangle=$ $=p(1-p)$-центральный момент второго порядка (дисперсия). Такие же соотношения справедливы для моментных функций $n$-ного порядка:

$$
K_{\lambda}^{n}\left(\vec{r}, \vec{r}_{1}, \vec{r}_{2}, \ldots\right)=D_{\lambda}^{n} f_{\lambda}^{n}\left(\vec{r}, \vec{r}_{1}, \vec{r}_{2}, \ldots\right),
$$

где $D_{\lambda}^{n}=(1-p)^{n} p+(-p)^{n}(1-p)$.

Порядок моментной функции и количество переменных, от которых она зависит, могут не совпадать. В таком случае моментные функции высших порядков могут быть выражены через моментные функции низших порядков:

$$
\begin{array}{r}
K_{\lambda}^{3}\left(\vec{r}, \vec{r}, \vec{r}_{1}\right)=(1-2 p) K_{\lambda}^{2}\left(\vec{r}, \vec{r}_{1}\right), \quad K_{\lambda}^{4}\left(\vec{r}, \vec{r}, \vec{r}, \vec{r}_{1}\right)=\left(1-3 D_{\lambda}^{2}\right) K_{\lambda}^{2}\left(\vec{r}, \vec{r}_{1}\right) \\
K_{\lambda}^{4}\left(\vec{r}, \vec{r}, \vec{r}_{1}, \vec{r}_{2}\right)=(1-2 p) K_{\lambda}^{3}\left(\vec{r}, \vec{r}_{1}, \vec{r}_{2}\right)+D_{\lambda}^{2} K_{\lambda}^{2}\left(\vec{r}_{1}, \vec{r}_{2}\right) \\
K_{\lambda}^{5}\left(\vec{r}, \vec{r}, \vec{r}_{1}, \vec{r}_{2}, \vec{r}_{3}\right)=(1-2 p) K_{\lambda}^{4}\left(\vec{r}, \vec{r}_{1}, \vec{r}_{2}, \vec{r}_{3}\right)+D_{\lambda}^{2} K_{\lambda}^{3}\left(\vec{r}_{1}, \vec{r}_{2}, \vec{r}_{3}\right)+p^{3} D_{\lambda}^{2} \\
K_{\lambda}^{5}\left(\vec{r}, \vec{r}_{1}, \vec{r}_{1}, \vec{r}_{2}, \vec{r}_{2}\right)=\left(1-4 p+4 p^{2}\right) K_{\lambda}^{3}\left(\vec{r}, \vec{r}_{1}, \vec{r}_{2}\right)+ \\
+p\left(1-3 p+2 p^{2}\right)\left[K_{\lambda}^{2}\left(\vec{r}, \vec{r}_{1}\right)+K_{\lambda}^{2}\left(\vec{r}, \vec{r}_{2}\right)\right]
\end{array}
$$

Моментные функции строятся для синтезированных объёмных структур. Процедура синтеза реализована с использованием различных алгоритмов и позволяет управлять такими параметрами, как разброс диаметров сфер (включений), сторона куба (выделенного объёма), объёмная доля включений. 
Значения моментной функции в зависимости от шага $\left|\vec{r}_{m}-\vec{r}_{n}\right|$ вычисляются с помощью следующей процедуры. Синтезированный фрагмент структуры разбивается сеткой с шагом $h=\omega d_{\min }$, где $d_{\min }$ - диаметр минимального включения. В узлах сетки проверяется наличие матрицы или включения, затем индикаторной функции (3) присваивается значение 0 или 1. Для получения значения моментной функции $n$-ного порядка (7) задаются расстояния $\left|\vec{r}_{m}-\vec{r}_{n}\right|$. Для всех пар узловых точек сетки, отстающих друг от друга на данные расстояния, вычисляются произведения $\lambda^{\prime}(\vec{r}) \lambda^{\prime}\left(\vec{r}_{1}\right) \cdots \lambda^{\prime}\left(\vec{r}_{n}\right)$ и делятся на количество таких пар узловых точек. Для вычисления следующего значения шаги пропорционально увеличиваются и процедура повторяется. При нулевом шаге точки радиус-векторов $\vec{r}, \vec{r}_{1}, \ldots, \vec{r}_{n}$ совпадают, следовательно, $\left\langle\lambda^{\prime}(\vec{r})\langle\lambda\rangle^{\prime}\left(\vec{r}_{1}\right) \cdots \lambda^{\prime}\left(\vec{r}_{n}\right)\right\rangle=\left\langle\lambda^{\prime}(\vec{r}) \lambda^{\prime}(\vec{r}) \cdots \lambda^{\prime}(\vec{r})\right\rangle=D_{\lambda}^{n}$. Таким образом, при нулевом шаге нормированные моментные функции $f_{\lambda}^{n}\left(\vec{r}, \vec{r}_{1}, \vec{r}_{2}, \ldots\right)$ всегда принимают значение, равное единице.

В настоящей работе при вычислении значений моментных функций все расстояния между точками принимаются одинаковыми: $\left|\vec{r}-\vec{r}_{1}\right|=\left|\vec{r}-\vec{r}_{2}\right|=$ $=\left|\vec{r}_{1}-\vec{r}_{2}\right|=\ldots=\left|\vec{r}_{m}-\vec{r}_{n}\right|$. Для построения моментных функций второго и четвертого порядков используется кубическая сетка (рис. 1, а), для функций третьего порядка - гексагональная сетка (рис. 1, б), моментные функции пятого порядка строятся на кубической центрированной сетке (рис. 1, в). При расчетах расстояние между узлами сетки бралось равным радиусу наименьшего включения $(\omega=0,5)$.

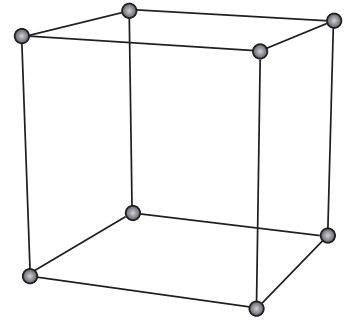

a

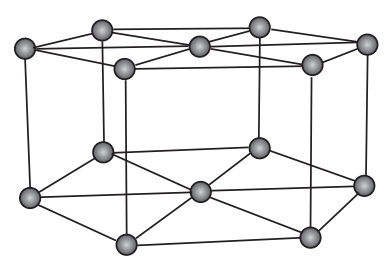

$\sigma$

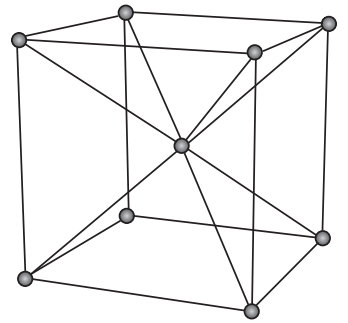

$B$

Рис. 1. Схематическое изображение ячеек сеток, используемых при построении моментных функций

3. Аппроксимация моментных функций. Для использования моментных функций при нахождении статистических характеристик полей деформирования в компонентах композитов необходимо знать их аналитический вид. Для того чтобы точнее подобрать единую аппроксимирующую зависимость для моментных функций одинакового порядка, но построенных для разных структур, можно нормировать переменную $\left|\vec{r}_{m}-\vec{r}_{n}\right|$ на величину, отражающую свойства структуры. Это позволит «приблизить» кривые функций друг к другу. В данной работе для нормирования была использована величина осреднённого минимального расстояния между включениями $d_{\text {avg }}$, которая вычисляется путем осреднения расстояний от центра каждой сферы (включения) до центра ближайшей к ней сферы.

В качестве универсального выражений для аппроксимации нормирован- 
ных моментных функций предлагается следующая зависимость:

$$
\begin{aligned}
f_{\lambda}^{n}\left(\vec{r}, \vec{r}_{1}, \vec{r}_{2}, \ldots\right)=\exp \left(-\frac{c_{1}}{d_{\text {avg }}} \sum_{i=0}^{n} \sum_{j=0}^{n} \mid \vec{r}_{i}-\right. & \left.\vec{r}_{j} \mid\right) \times \\
& \times \cos \left(\frac{c_{2}}{d_{\text {avg }}^{2}} \sum_{i=0}^{n} \sum_{j=0}^{n}\left|\vec{r}_{i}-\vec{r}_{j}\right|^{2}\right),
\end{aligned}
$$

где $n$ - порядок функции.

В настоящей работе использовались синтезированные структуры с параметрами, указанными в табл. 1. Радиусы включений (сфер) распределены по равномерному закону. Величины, характеризующие радиус и расстояние, указаны в безразмерных условных единицах.

Коэффициенты аппроксимирующего выражения вычисляются методом смешанных градиентов. На рис. 2 представлено сравнение графиков моментных функций, построенных на сетке для структуры № 2, с графиками аппроксимирующих выражений для этих функций. Коэффициенты выражения (8) можно найти в табл. 2.

Наибольшее отклонение графика аппроксимирующего выражения наблюдается при построении моментной функции 2-го порядка, но при это максимальная величина не превышает 0,05. Для моментных функций 3-го, 4-го и 5-го порядков максимальная величина отклонения составляет около 0,02, поэтому графики практически совпадают. Как видно, выражение (8) с достаточной точностью аппроксимирует моментные функции, а следовательно, может быть использовано для аналитических вычислений.

Графики нормированных многоточечных моментных функций второго, третьего и четвертого порядков для структур с параметрами, указанными в

\begin{tabular}{c|c|c|c|c|c}
\hline & $\begin{array}{c}\text { Объёмная } \\
\text { доля, } p\end{array}$ & $\begin{array}{c}\text { Кол-во } \\
\text { вклю- } \\
\text { чений, } N\end{array}$ & $\begin{array}{c}\text { Минималь- } \\
\text { ный радиус } \\
\text { включений }\end{array}$ & $\begin{array}{c}\text { Максималь- } \\
\text { ный радиус } \\
\text { включений }\end{array}$ & $\begin{array}{c}\text { Величина } \\
d_{\text {avg }}\end{array}$ \\
\hline Структура № 1 & 0,20 & 1047 & 4 & 12 & 18,9941 \\
Структура № 2 & 0,25 & 1193 & 4 & 16 & 18,2389 \\
Структура № 3 & 0,30 & 976 & 4 & 28 & 19,5464 \\
\hline
\end{tabular}

Таблица 2

\begin{tabular}{c|l|c|c|c|c}
\hline \multicolumn{2}{l|}{} & $\begin{array}{c}\text { Функция } \\
\text { 2-го порядка }\end{array}$ & $\begin{array}{c}\text { Функция } \\
\text { 3-го порядка }\end{array}$ & $\begin{array}{c}\text { Функция } \\
\text { 4-го порядка }\end{array}$ & $\begin{array}{c}\text { Функция } \\
\text { 5-го порядка }\end{array}$ \\
\hline Структура № 1 & $c_{1}$ & 3,1762 & 4,1242 & 4,7187 & 4,9281 \\
$(p=0,20)$ & $c_{2}$ & 3,2175 & 3,1281 & 3,8203 & 3,9610 \\
Структура № 1 & $c_{1}$ & 3,1128 & 3,8509 & 4,2839 & 4,5152 \\
$(p=0,25)$ & $c_{2}$ & 2,4707 & 2,0138 & 2,5263 & 2,6647 \\
Структура № 3 & $c_{1}$ & 2,9704 & 3,5150 & 4,0205 & 4,2141 \\
$(p=0,30)$ & $c_{2}$ & 1,8648 & 1,2450 & 1,5240 & 1,5690 \\
\hline
\end{tabular}



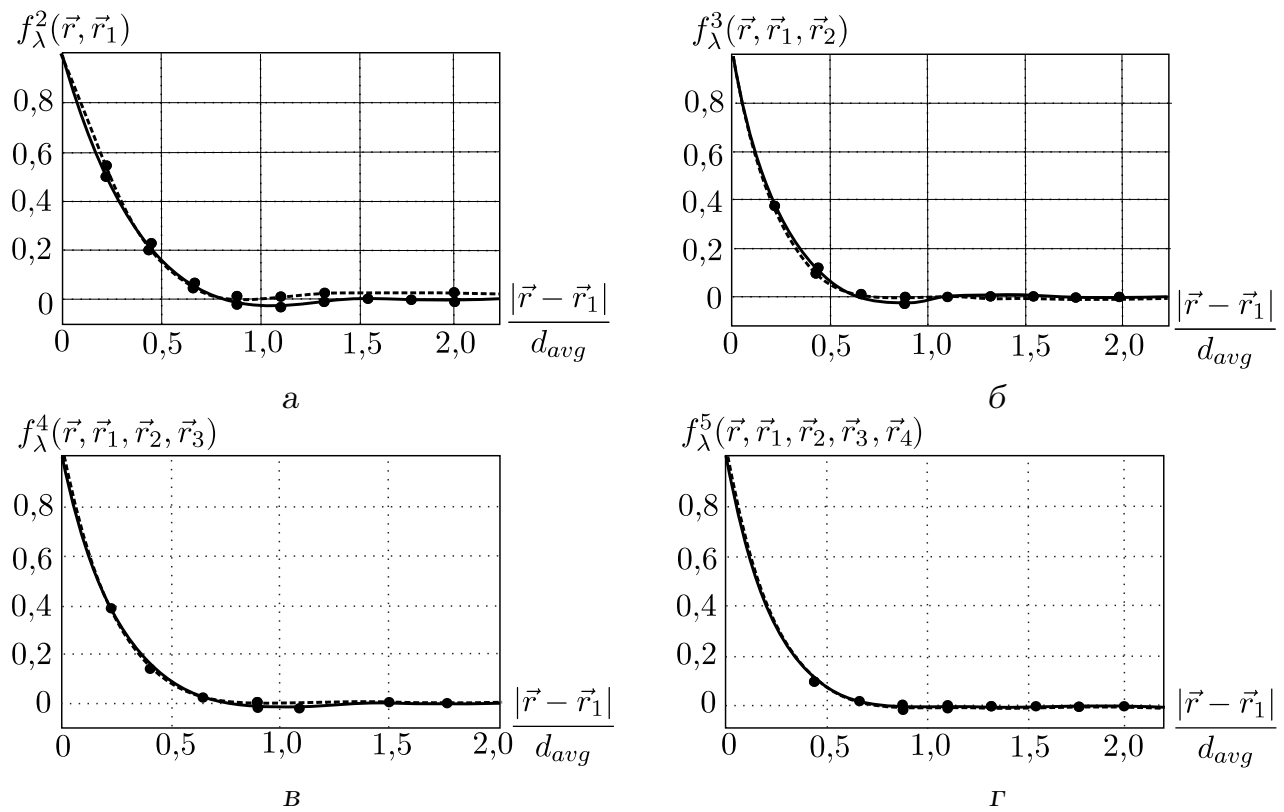

Рис. 2. Сравнение графиков моментных функций: сплошная линия - график моментной функции, построенной по сетке; штриховая линия - график аппроксимирующего выражения; а) моментная функция 2-го порядка, б) моментная функция 3-го порядка, в) моментная функция 4-го порядка, г) моментная функция 5-го порядка

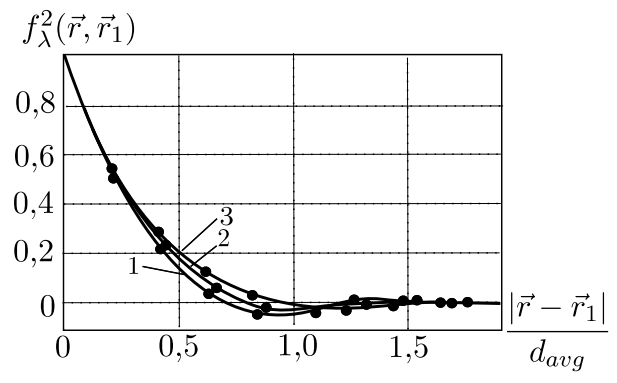

$f_{\lambda}^{4}\left(\vec{r}_{,} \vec{r}_{1}, \vec{r}_{2}, \vec{r}_{3}\right)$

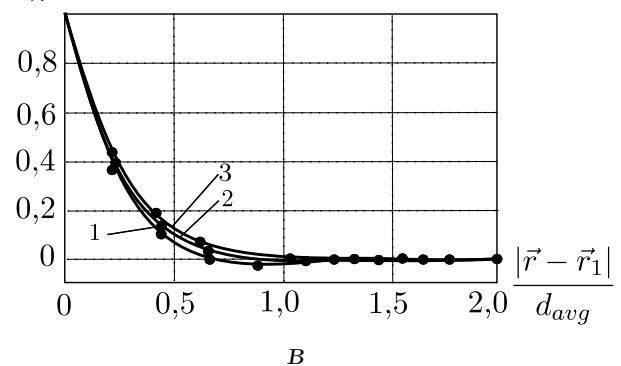

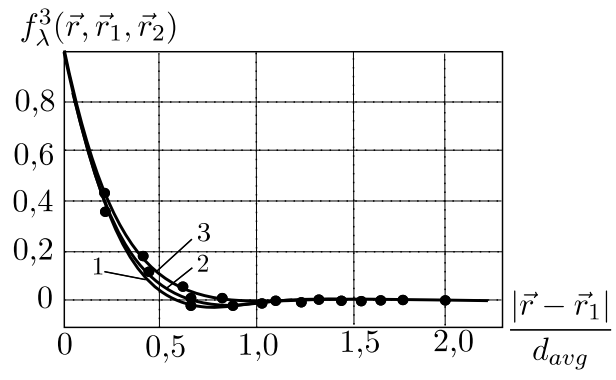

б

$f_{\lambda}^{5}\left(\vec{r}, \vec{r}_{1}, \vec{r}_{2}, \vec{r}_{3}, \vec{r}_{4}\right)$

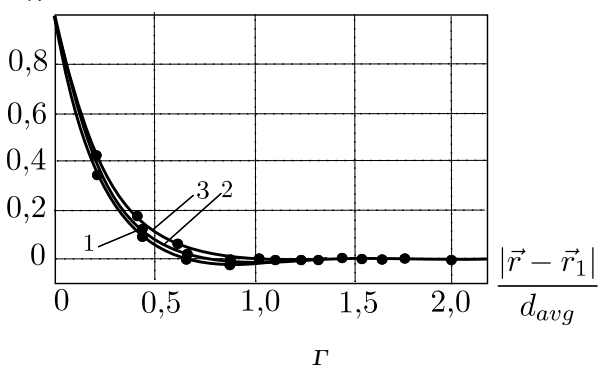

Рис. 3. Графики аппроксимирующих выражений многоточечных нормированных моментных функций для структур с различной объёмной долей: а) 2-го порядка, б) 3-го порядка, в) 4-го порядка, г) 5-го порядка; 1) $p=0,2,2) p=0,25,3) p=0,3$ 
табл. 1, аппроксимированных выражением (8), представлены на рис. 3. Коэффициенты выражения (8) вынесены в табл. 2.

Выводы. На основании исследований моментных функций индикаторной функции $\lambda(\vec{r})$ можно сделать два важных вывода. Первый состоит в том, что моментные функции являются локальными. Область затухания моментных функций соответствует области статистической зависимости, т. е. области, в которой значения моментных функций отличны от нуля. Если моментные функции быстро затухают, то считается, что в расположении элементов структуры имеет место ближний порядок [5]. Это означает, что на формирование полей деформирования в некоторой области, содержащей произвольно выделенное включение, решающее влияние оказывают лишь ближайшие к нему включения из их произвольного большого множества. Второй вывод заключается в том, что моментные функции имеют область отрицательных значений, что свидетельствует о наличии периодических составляющих в этих случайных полях [9].

Таким образом, рассмотрен способ решения стохастической краевой задачи теории упругости с использованием моментных функций структурных свойств. Описан алгоритм построения моментных функций $n$-ного порядка на различных типах сетки. Приведено универсальное аналитическое выражение для аппроксимации моментных функций высших порядков. Для ряда полидисперсных структур получены графики и коэффициенты аппроксимирующего выражения.

Работа выполнена при поддержке РФФИ (проект № 11-01-96030-р_урал_а).

\section{БИБЛИОГРАФИЧЕСКИЙ СПИСОК}

1. Buryachenko V.A. Micromehcanics of heterogenous materials. New York: Springer-Verlag, 2007. 687 pp.

2. Лифшич И. М., Розенцвейг Л. Н. К теории упругих свойств поликристаллов // ЖЭТФ, 1946. T. 16, № 11. C. 967-980. [Lifshits I. M., Rozentsveig L. N. Theory of elastic properties of polycrystals // ZhETF, 1946. Vol. 16, no.11. Pp. 967-980].

3. Волков С.Д., Ставров В. П. Статистическая механика композиционных материалов. Минск: БГУ, 1978. 206 с. [Volkov S.D., Stavrov V.P. Statistical mechanics of composite materials. Minsk: BGU, 1978. 206 pp.]

4. Ломакин В. А. Статистические задачи механики твердых деформируемых тел. М.: Наука, 1970. 139 с. [Lomakin V. A. Statistical problems of the mechanics of solid deformable bodies. Moscow: Nauka, 1970. 139 pp.]

5. Шермергор Т. Д. Теория упругости микронеоднородных материалов. М.: Наука, 1977. 400 c. [Shermergor T. D. Theory of elasticity of microheterogeneous media. Moscow: Nauka, 1977. 400 pp.]

6. Соколкин Ю.В., Ташкинов А.А. Механика деформирования и разрушения структурно неоднородных тел. М.: Наука, 1984. 116 с. [Sokolkin Yu. V., Tashkinov A.A. Mechanics of deformation and dracture of structurally inhomogeneous bodies. Moscow: Nauka, 1984. 116 pp.]

7. Панъков А.А. Статистическая механика пьезокомпозитов. Пермь: ПГТУ, 2009. 480 с. [Pankov A. A. Statistical mechanics of piezocomposites. Perm: PGTU, 2009. 480 pp.]

8. Ташкинов М.А., Вилъдеман В.Э., Михайлова Н. В. Метод последовательных приближений в стохастической краевой задаче теории упругости структурно-неоднородных сред// Механика композичионных материалов и конструкций, 2010. Т.16, №3. C. 369-383; англ. пер.: Tashkinov M.A., Vil'deman V.E., Mikhailova N. V. Method of 
successive approximations in a stochastic boundary-value problem in the elasticity theory of structurally heterogeneous media// Composites: Mechanics, Computations, Applications, An International Journal, 2011. Vol. 2, no. 1. Pp. 21-37.

9. Christensen R.M. Mechanics of composite materials. New York: Willey-Interscience, 1979. 348 рр.; русск. пер.: Кристенсен Р. Введение в механику композитов. М.: Мир, 1984. $336 \mathrm{c.}$

Поступила в редакцию 25/III/2011;

в окончательном варианте - 16/V/2011.

MSC: 74A40, 74B20

\section{MULTIPOINT MOMENT FUNCTIONS OF STRUCTURAL PROPERTIES FOR POLYDISPERSE COMPOSITES}

\section{A. Tashkinov}

Perm State Technical University,

29a, Komsomolskiy prospekt, Perm, Russia, 614990.

E-mail: m.tashkinov@mail.ru

The stochastic boundary-value problem of elasticity theory for two-phase polydisperse composites is stated. The solution method with using high order moment functions is described. Algorithm of synthesis of n-order moment functions for $3 D$ structures is presented. Approximating expression for moment functions is suggested. Examples of calculation of high-order moment functions for polydisperse structures are given.

Key words: composites, moment functions, random polydisperse structure, $3 D$ models, boundary-value problem, approximation.

Original article submitted 25/III/2011; revision submitted $16 / \mathrm{V} / 2011$.

Mikhail A. Tashkinov, Postgraduate Student, Dept. of Mechanics of Composition Materials $\&$ Structures. 